\title{
Sensing and Sharing Schemes for Spectral Efficiency of Cognitive Radios
}

\author{
M. K. Kaushik, Y. Yoganandam, S. K. Sahoo
}

Department of Electrical Engineering, BITS Pilani Hyderabad Campus, India

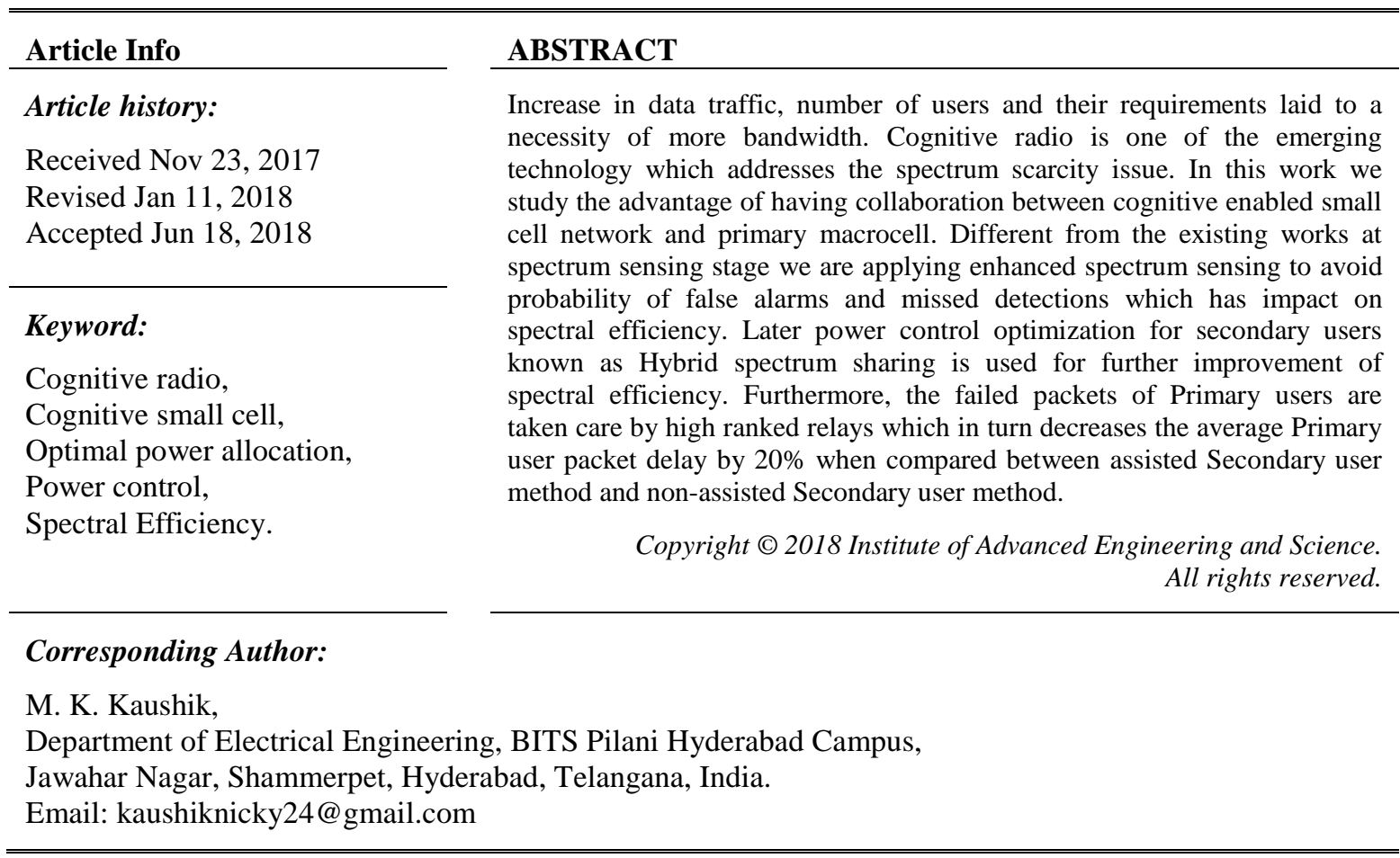

\section{INTRODUCTION}

Day by day increase in data traffic and widespread usage of smart mobile devices are demanding more bandwidth. Survey reveals that the allocated spectrum is extremely underutilized in temporal and spectral domain [1]. The problems which are encountered between demand growth and underutilization of spectrum are addressed by the introduction of Cognitive radios. The foremost feature of Cognitive radio is to enhance the spectrum efficiency by letting secondary (unlicensed) users utilize the Primary (Licenced) bands opportunistically without causing interference.

In cognitive radio environment the secondary users sense the primary user bands and based on the decision they access the spectrum for their communication. Most commonly used spectrum access models [3],[5],[6],[13],[20] are interweave mode and joint interweave underlay mode. In the interweave mode secondary users transmit the information when primary users are sensed to be idle. Due to sensing errors primary user may get interfered by secondary user transmission. In the joint interweave underlay mode, secondary users will transmit the information irrespective of primary user transmission, but without interfering and maintaining the Quality of service of primary users.

To have interference free spectrum access a reliable spectrum sensing method must be chosen and also to avoid false alarms and missed detections. Good amount of spectrum sensing algorithms have been discussed in the survey to recognise the presence of primary user transmissions [8],[9],[10]. There are tradeoffs in existing solutions like required sensing time, detection capabilities and complexity. In general a secondary user is not likely to have any prior knowledge about primary signals that are present in particular frequency band. In these scenarios the energy detection method is used because of its capability to work without having any prior knowledge about the signal to be detected. Regardless of its practical limitations it is been chosen because of its simplicity, low implementation costs and computations. But the conventional 
energy detection has increase in the probability of false alarms and missed detections. To overcome an Enhanced Energy detector is proposed in [11].

In 5G networks [12] cognitive radio is one among the emerging technologies to improve the spectrum access efficiently. Small cell technology is promising in 5G networks because it can offload traffic from primary marcocells and it can increase the spatial reuse and coverage. By having cognitive small cell deployments in the network resource allocation, spectrum access and interference mitigation are maintained optimally [13-19]. Therefore system performance can further be improved by having small cell network coexisting with a macro cell network [20]. The three potential ways of sharing primary macrocell spectrum by small cell network are: 1) Spectrum sharing, primary macrocells spectrum is shared by small cell 2) opportunistic spectrum access, secondary small cell can access the primary macro cell spectrum if it is sensed idle 3) Hybrid spectrum sharing, depending on the spectrum sensing result of Enhanced spectrum sensing secondary small cell senses channel status and optimize the allocation of power [21].

In [22], for perfect sensing case authors studied throughput tradeoff between only interweave and combination of interweave underlay mode. In [22]-[25] authors hasn't considered any cooperation between users transmission. In [26]-[28] under information theoretic framework authors discussed about benefits in cooperation between users. In [29] SU's receiving packets from two PU's relaying them using superposition coding method when PU is idle. In [30] cooperative relaying is discussed where the failed packets of PU are transmitted by SU relays which has finite queue length. Coming to survey on sharing techniques in [31] a theoretically derived stochastic geometry model was investigated for spectral sharing scheme between macrocell (Primary network) and small cell (Secondary network). In [32], a stochastic dual control approach was presented to analyse different interfering forces within small cell networks, without involving centralized and global control efforts. In [33], a stackleberg game in cognitive femto cells was studied for energy efficient power allocation and spectrum sharing. A power adaptation game was studied to decrease energy consumption in [34]. The existing works discussed so far used conventional energy detectors at sensing stage due to which false alarms and missed detections will increase and has not been well tested on improvement of spectral efficiency by having power control optimization.

In this paper a combination of Enhanced spectrum sensing (ESS) and hybrid spectrum sharing (HSS) are used for enhancing the primary and secondary user spectral efficiency. Spectrum sensing is done by ESS and based on the sensing result cognitive small cell(CSC) optimizes the power allocation by using HSS. In recent times the efforts are towards secondary network helping to relay primary user packets by continuing data transfer of their own.This is motivated because of joint interweave underlay mode. With various constraints and objectives, extensive research is done in the literature [2]-[7]. Hence we considered the influence of having a group of buffered relay nodes with cognitive capabilities on Primary and secondary user Spectral efficiency. Relay nodes will help in delivering unsuccessful packets at primary destination nodes. When both primary and secondary nodes are silent the relay nodes will send the unsuccessful packets.

\section{SYSTEM MODEL}

In this work we considered an OFDMA based cognitive enabled small cell network. In OFDMA system $\mathrm{N}$ subchannels are divided from a total bandwidth of $\mathrm{B}$ and is assumed that within a subchannel, channel fading of each subcarrier is similar, but differ across different subchannels. The main focus of our work is optimal power allocation in downlink of secondary small cell based on spectrum sensing result and set of SU relays helping PU to transmit their failed packets. Cognitive small cell perform ESS on primary macro cells to find the status of subchannels. Based on the sensing result CSC adapts the transmit power.

\subsection{Enhanced Spectrum Sensing}

One of the prime challenges of cognitive radio network is it should not cause any kind of harmful interference to primary users. In order to have interference free network secondary user or cognitive radio must identify the presence of primary user reliably. To do so we are applying enhanced spectrum sensing which has low probability of false alarms and missed detections compared to conventional energy detectors.The probability of detection and false alarms [11] are given as below. The threshold for a target probability of false alarms [35] is given by

$$
\lambda=\left(Q^{-1}\left(P_{\text {fa,target }}^{C E D}\right) \sqrt{2 N}+N\right) \sigma_{\omega}^{2}
$$

Then the ESS [36] theoretic performance may be characterized by 


$$
\begin{aligned}
& \begin{aligned}
P_{d}^{E E D} & =P\left\{T\left(y_{i}\right)>\lambda\right\}_{\mathcal{H}_{1}}+P\left\{T\left(y_{i}\right) \leq \lambda\right\}_{\mathcal{H}_{1}} \cdot P\left\{T^{a v g}\left(T_{i}\right)>\lambda T\left(y_{i}\right) \leq \lambda\right\}_{\mathcal{H}_{1}} \\
P_{f a}^{E E D} & =P\left\{T\left(y_{i}\right)>\lambda\right\}_{\mathcal{H}_{0}}+P\left\{T\left(y_{i}\right) \leq \lambda, T^{a v g}\left(T_{i}\right) \lambda\right\}_{\mathcal{H}_{0}} \\
& =P\left\{T\left(y_{i}\right)>\lambda\right\}_{\mathcal{H}_{0}}+P\left\{T\left(y_{i}\right) \leq \lambda\right\}_{\mathcal{H}_{0}}
\end{aligned} \\
& \cdot P\left\{T^{a v g}\left(T_{i}\right)>\lambda T\left(y_{i}\right) \leq \lambda\right\}_{\mathcal{H}_{0}} \\
& P_{d}^{E E D} \simeq P_{d}^{C E D}+\left(1-P_{d}^{C E D}\right) \mathcal{Q}\left(\frac{\lambda-\mu_{\text {avg }}}{\sigma_{\text {avg }}}\right) \\
& P_{f a}^{E E D} \simeq P_{f a}^{C E D}+\left(1-P_{f a}^{C E D}\right) \mathcal{Q}\left(\frac{\lambda-\mu_{\text {avg }}}{\sigma_{\text {avg }}}\right)
\end{aligned}
$$

Where, $P_{d}^{E E D}$ is the probability of detection using the ESS, $T^{a v g}$ is the average time for detection, $P_{f a}^{E E D}$ is the probability of false alarms using the ESS. $P_{d}^{C E D}$ and $P_{f a}^{C E D}$ are the probability of detection and false alarms using the classical energy detection. $\mu_{a v g}$ and $\sigma_{a v g}$ are the mean the variance of the samples [37].

\subsection{Hybrid Spectrum Sensing}

As shown in the figure below [38] the frame structure of cognitive small cell network is designed. It is observed from the figure that a sensing time $\tau$ is assigned in the beginning of each frame. The cognitive small cell (CSC) adapts it's transmit power at the beginning of each frame dependent on the sensing result.

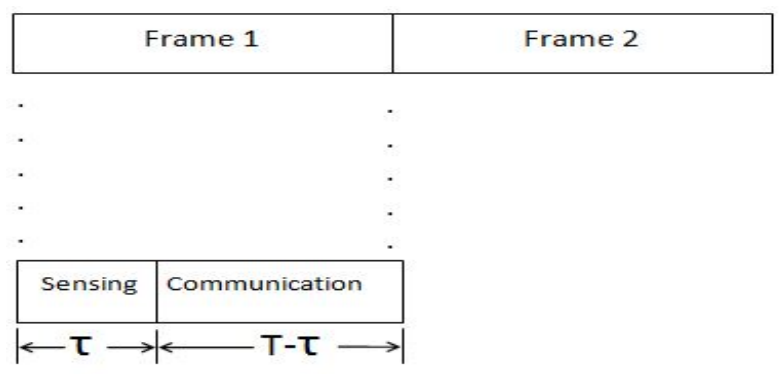

Frame $\mathrm{N}$

Figure 1. Frame structure of CSC network

If the subchannel is detected idle, CSC can transmit with high power $\mathrm{P}_{\mathrm{s}, \mathrm{n}}^{\mathrm{v}}$ : if not CSC can transmit with low power $\mathrm{P}_{s, \mathrm{n}}^{0}$. This method is termed as "Hybrid Spectrum Sharing". Based on shannons capacity formulae the feasible capacity of subchannel $n$ when sensing outcome is idle in small cell is given by

$$
R_{v, n}=\log _{2}\left(1+\frac{g_{s s, n} \cdot p_{s, n}^{v}}{\sigma^{2}}\right)
$$

Where $g_{s s, n}$ is the channel gain between macrocell base station (MBS) and CSC of subchannel n. The capacity on subchannel $\mathrm{n}$ in CSC when sensing result is occupied/active is given as

$$
R_{o, n}=\log _{2}\left(1+\frac{g_{s s, n} \cdot p_{s, n}^{0}}{g_{m s, n} \cdot p^{0}{ }_{m, n}+\sigma^{2}}\right)
$$

Where $g_{m s, n}$ is the channel gain and $p^{0}{ }_{m, n}$ is the transmit power on subchannel $\mathrm{n}$.

\subsection{Formulation of Interference and Transmit Power Constraints}

In this section we are trying to determine the optimal powers of CSC's which overcome the interference mitigation. From [33],[39],[40] average interference and transmit powers are considered and can be formulated as follows 


$$
\begin{aligned}
& E_{g, h}\left\{p\left(H_{0}\right)\left(1-p_{f a}\right) p+p\left(H_{1}\right)\left(1-p_{d}\right) p\right\} \leq p_{a v} \\
& E_{g, h}\left\{p\left(H_{1}\right)\left(1-p_{d}\right) h p\right\} \leq \Gamma
\end{aligned}
$$

Where $p_{a v}$ the maximum average is transmit power of CSC users and $\Gamma$ is the maximum average interference power which can be tolerated by PU. The enhanced spectrum sensing is considered as method of detection, the detection and false alarm probability are given by Equation 8 and Equation 9. Now the formulation for average interference and transmit power constraint of CSC users for the combination of Hybrid spectrum sharing and enhance spectrum sensing is given as

$$
\begin{aligned}
& \operatorname{maximize}\left\{p, p_{d}^{E E D}\right\} ; C\left(p, p_{d}^{E E D}\right) \\
& =E_{g, h}\left\{p\left(H_{0}\right)\left(1-p_{f a}^{E E D}\right) \cdot \log _{2}\left(1+\frac{g p}{\sigma^{2}}\right)+p\left(H_{1}\right)\left(1-p_{d}^{E E D}\right) \cdot \log _{2}\left(1+\frac{g p}{\sigma^{2}{ }_{n}+\sigma^{2}{ }_{p}}\right)\right\}
\end{aligned}
$$

Where $\mathrm{g}$ and $\mathrm{h}$ are instantaneous channel gains. $\sigma_{n}^{2}$ and $\sigma^{2}{ }_{p}$ are variance of noise and signal. $p\left(H_{0}\right)$ and $p\left(H_{1}\right)$ are probability of PU present or not present. By letting similar average interference power constraint in cognitive networks [41], the $p_{d}^{E E D}$ becomes an optimization variable for maximizing the achievable spectral efficiency. By observing Equation 10 it is evident that the formulation is convex with transmit power $\mathrm{P}$ but not with respect to $p_{d}^{E E D}$, because of dependency on probability of false alarm $p_{f a}{ }^{E E D}$ on the probability of detection [42].

Hence, the optimal detection probability cannot yield from convex optimization techniques. In this case for minimization of spectral efficiency of the proposed HSS and ESS we should consider the detection probability is in the range [0,1]. By doing so the optimal power allocation can be determined for a detection probability $p_{d}=p_{d}^{E E D}$. Lagrangian can be determined for detection probability $p_{d}=p_{d}{ }^{E E D}$ in accordance with transmit power $\mathrm{P}$ is given by

$$
\begin{gathered}
L(p, \lambda, \mu)=E_{g, h}\left\{p\left(H_{0}\right)\left(1-p_{f a}{ }^{E E D}\left(\mathbb{P}_{d}^{E E D}\right)\right) \cdot \log _{2}\left(1+\frac{g p}{\sigma^{2}}\right)\right. \\
\left.+p\left(H_{1}\right)\left(1-\mathbb{P}_{d}^{E E D}\right) \cdot \log _{2}\left(1+\frac{g p}{\sigma^{2}{ }_{n}+\sigma^{2}}\right)\right\} \\
-\lambda\left\{E_{g, h}\left[p \cdot p\left(H_{0}\right)\left(1-p_{f a}^{E E D}\left(\mathbb{P}_{d}{ }^{E E D}\right)\right)+p\left(H_{1}\right)\left(1-\mathbb{P}_{d}{ }^{E E D}\right) p\right]-p_{a v}\right\} \\
-\mu\left\{E_{g, h}\left[p\left(H_{1}\right)\left(1-\mathbb{P}_{d}\right) h p\right]-\Gamma\right\}
\end{gathered}
$$

Now the lagrange dual optimization problem is given as

$$
\text { minimize } \lambda \geq 0, \mu \geq 0, \quad g(\lambda, \mu)
$$

Where $g(\lambda, \mu)$ is lagrange dual function and given as

$$
g(\lambda, \mu)={ }_{P}^{S U P} L(p, \lambda, \mu)
$$

From [42] the primal optimization problem (Eq 10) can be solved by Equation 12 with respect to transmit power P. Hence our focus is to solve lagrange dual optimization problem Equation 10. The Supremum of lagrangian $L(p, \lambda, \mu)$ is to find first to calculate the lagrange dual function $g(\lambda, \mu)$ with respect to transmit power $\mathrm{P}$ as in Equation 13. With the help of KKT conditions [42] the optimal power allocation $\mathrm{P}$ for multipliers $\lambda$ and $\mu$ can be obtained by

$$
p=\left[\frac{A+\sqrt{\Delta}}{2}\right]^{+}
$$

The parameters A and $\Delta$ are given as follows and the whole term $[\mathrm{X}]^{+}$denotes $\max (\mathrm{o}, \mathrm{x})$

$$
A=\frac{\log _{2} e\left[p\left(H_{0}\right)\left(1-p_{f a}{ }^{E E D}\left(\mathbb{P}_{d}{ }^{E E D}\right)\right)+p\left(H_{1}\right)\left(1-\mathbb{P}_{d}^{E E D}\right)\right]}{\lambda\left[p\left(H_{0}\right)\left(1-p_{f a}{ }^{E E D}\left(\mathbb{P}_{d}{ }^{E E D}\right)\right)+p\left(H_{1}\right)\left(1-\mathbb{P}_{d}{ }^{E E D}\right)\right]+\mu p\left(H_{1}\right)\left(1-\mathbb{P}_{d}{ }^{E E D}\right) h}-\frac{2 \sigma^{2}{ }_{n}+\sigma^{2} p}{g}
$$




$$
\Delta=A^{2}+\frac{4}{g}\left\{\frac{\log _{2} e\left[p\left(H_{0}\right)\left(1-p_{f a}{ }^{E E D}\left(\mathbb{P}_{d}{ }^{E E D}\right)\right)\left(\sigma^{2}{ }_{n}+\sigma^{2} p\right)+p\left(H_{1}\right)\left(1-\mathbb{P}_{d}{ }^{E E D}\right) \sigma^{2}{ }_{n}\right]}{\lambda\left[p\left(H_{0}\right)\left(1-p_{f a}\left(\mathbb{P}_{\boldsymbol{d}}\right)\right)+p\left(H_{1}\right)\left(1-\mathbb{P}_{d}\right)\right]+\mu p\left(H_{1}\right)\left(1-\mathbb{P}_{d}{ }^{E E D}\right) h}-\frac{\sigma^{2}{ }_{n}\left(\sigma^{2}{ }_{n}+\sigma^{2} p\right)}{g}\right\}
$$

\section{RESULTS AND ANALYSIS}

In this section to evaluate the performance of the proposed method we are going to present the simulation results of the network consisting of cognitive small cell and macrocell networks. The comparison results are between HSS+ESS, OSS+ESS [40] and CSS [5] [6]. From the following results it is evident that by having optimal powers in Hybrid spectrum sharing [21] with the involvement of Enhanced energy detection [11] will yield better results. The parameters considered are, sampling frequency, $\mathrm{f}=6 \mathrm{MHz}, \mathrm{T}=0.1$ sec, and number of nodes in the network, $\mathrm{N}=50$, variance $\sigma^{2}=1 \times 10^{-4}$ and channel gains are considered as block faded and distributed exponentially with 0.1 mean. On each channel of primary macrocell the transmit power is set as $10 \mathrm{~mW}$. The assumptions are, the detection probability is considered as $90 \%$ if it is not stated and $0.3 \mathrm{bps} / \mathrm{Hz}$ is the minimum data requirement for better Quality of Service. As it can be seen from Figure 2 by having efficient energy detection scheme at sensing unit and optimal power allocation for secondary users in Hybrid spectrum sharing will eventually increase spectral efficiency when compared with Opportunistic [38] and Conventional Spectrum sensing [5] [6] in the environment with parameters considered above. One more reason for increment in spectral efficiency is the failed packets of PU are taken care by a set of high ranked SU relays. Hence the Spectral efficiency of PU has increased compared to other methods [25] [26] [27] [28].

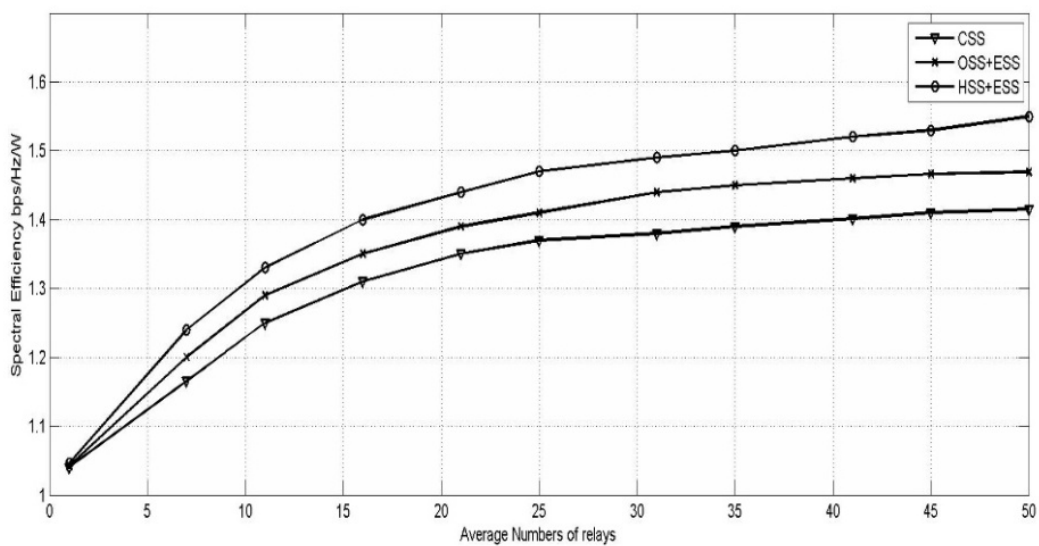

Figure 2. Comparison of average number of relays and its impact on PU Spectral Efficiency between different methods

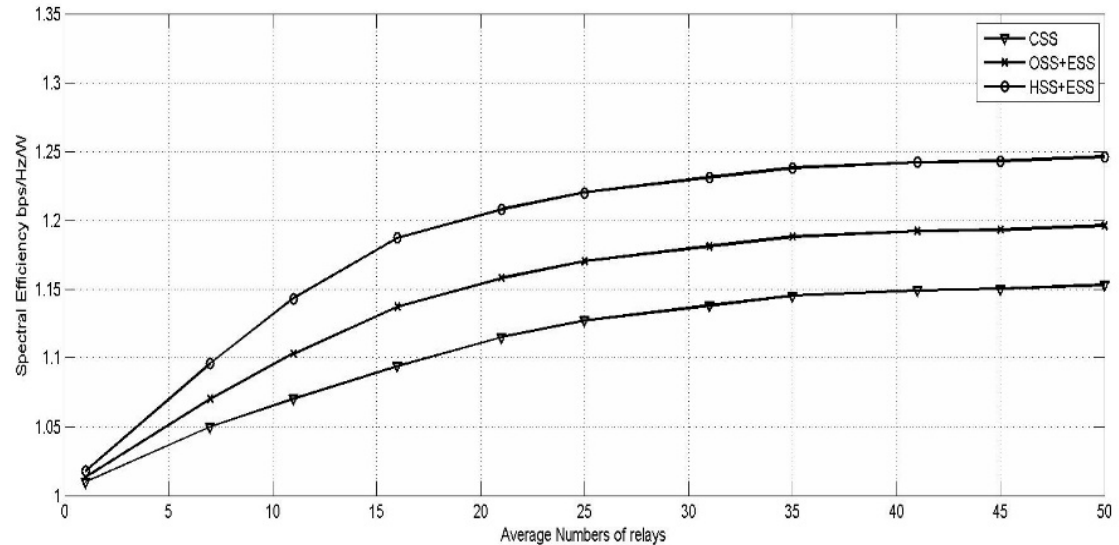

Figure 3. Comparison of average number of relays and its impact on SU Spectral Efficiency between different methods 
Figure 3 displays that by choosing optimal powers to SU's and increase in the number of relays will increase its spectral efficiency without causing interference to PU's. If we observe the SU's who opportunistically access the PU spectrum (CSS) [5] [6] with conventional energy detector and Enhanced energy detector (OSS+ESS) [40] have low spectral efficiency compared to the proposed method (HSS+ESS) because of not assigning optimal powers

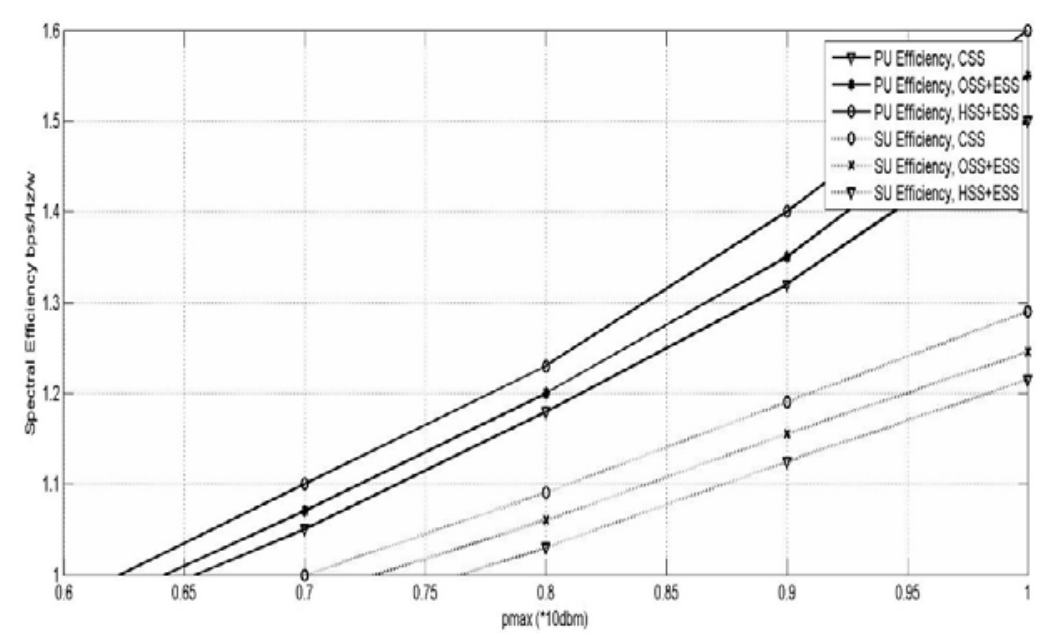

Figure 4. Spectral Efficiency vs. Pmax (*10dbm) in both Primary macrocell and Cognitive small cell

Figure 4 provides the spectral efficiency comparison between proposed method and the other methods of primary macrocell and cognitive small cell. By implementing the proposed method and working in complete environment where primary macrocell and cognitive small cell nodes are pariticipating in communication. The spectral efficiency has increased in both the networks by having Hybrid spectrum sharing plus enhanced energy detection and SU relay nodes helping PU failed packet transmission.

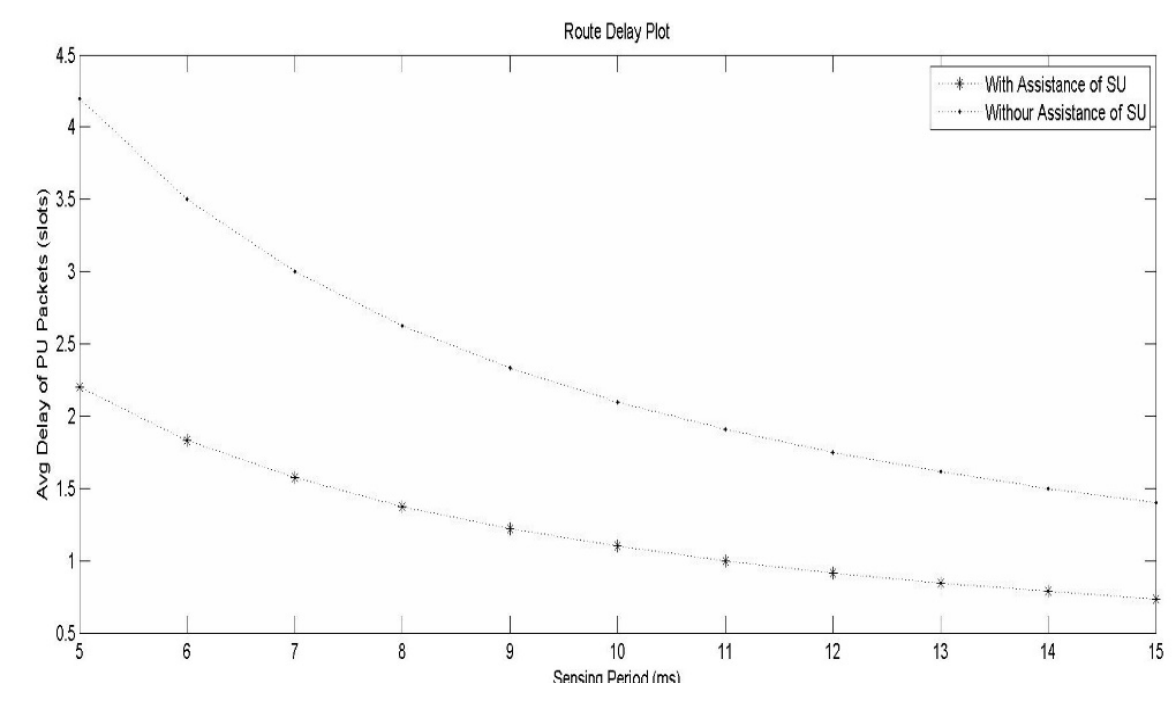

Figure 5. Average Delay of PU packets with respect to time with and without assistance of Cognitive small cell relays

Figure 5 indicates the relation between Sensing Time and average delay of PU packets with and without assistance of SU relays. One of our objective was if any failed packets are there from PU, the high ranked SU relays in cognitive small cell will help PU's to transfer the packets. By doing so it is observed from figure 5 that the average delay of PU packets has decreased by $20 \%$. Hence we can say that our 
proposed scheme's spectral efficiency is better when compared with other methods and the average packet delay is also decreased.

\section{CONCLUSION}

In this work, we proposed a method which allocates an optimal power to SU's in order to mitigate interference for PU's. We also have investigated a cooperation method where a cognitive small cell nodes (SU's) will help Primary macro cell nodes (PU's) in transmission of failed packets. We analysed spectral efficiencies and average PU packet delay. It was observed from the results that the proposed method's spectral efficiency is improved for primary macrocell and cognitive small cell. The average PU packet delay of assisted SU method is decreased by $20 \%$ when compared to non assisted SU method.

\section{REFERENCES}

[1] McHenry MA. NSF spectrum occupancy measurements project summary. Shared spectrum company report. 2005 Aug 15.

[2] Tragos EZ, Zeadally S, Fragkiadakis AG, Siris VA. Spectrum assignment in cognitive radio networks: A comprehensive survey. IEEE Communications Surveys \& Tutorials. 2013 Jan 1; 15(3): 1108-35.

[3] Gaafar M, Amin O, Abediseid W, Alouini MS. Underlay spectrum sharing techniques with in-band full-duplex systems using improper Gaussian signaling. IEEE Transactions on Wireless Communications. 2017 Jan; 16(1): 235-49.

[4] Feng D, Jiang C, Lim G, Cimini LJ, Feng G, Li GY. A survey of energy-efficient wireless communications. IEEE Communications Surveys \& Tutorials. 2013 Mar; 15(1): 167-78.

[5] Amin O, Abediseid W, Alouini MS. Overlay spectrum sharing using improper Gaussian signaling. IEEE Journal on Selected Areas in Communications. 2017 Jan; 35(1): 50-62.

[6] Ozcan G, Gursoy MC. Optimal power control for underlay cognitive radio systems with arbitrary input distributions. IEEE Transactions on Wireless Communications. 2015 Aug; 14(8): 4219-33.

[7] Pei Y, Liang YC, Teh KC, Li KH. Energy-efficient design of sequential channel sensing in cognitive radio networks: optimal sensing strategy, power allocation, and sensing order. IEEE Journal on Selected Areas in Communications. 2011 Sep; 29(8): 1648-59.

[8] Ariananda DD, Lakshmanan MK, Nikookar H. A survey on spectrum sensing techniques for cognitive radio. InCognitive Radio and Advanced Spectrum Management, 2009. CogART 2009. Second International Workshop on 2009 May 18 (pp. 74-79). IEEE.

[9] Ali SS, Liu C, Jin M. Minimum Eigenvalue Detection for Spectrum Sensing in Cognitive Radio. International Journal of Electrical and Computer Engineering. 2014 Aug 1; 4(4): 623.

[10] Amini M, Mirzavandi A, Rezaei M. Discrete Markov chain based spectrum sensing for cognitive radio. International Journal of Electrical and Computer Engineering. 2015 Apr 1; 5(2): 297.

[11] Kaushik MK, Sai TS, Yoganandam Y. Enhanced energy detection and moving average thresholding for cognitive femtocell networks. InMicroelectronics and Electronics (PrimeAsia), 2013 IEEE Asia Pacific Conference on Postgraduate Research in 2013 Dec 19 (pp. 250-253). IEEE.

[12] Jiang C, Zhang H, Ren Y, Chen HH. Energy-efficient non-cooperative cognitive radio networks: micro, meso, and macro views. IEEE Communications Magazine. 2014 Jul; 52(7): 14-20.

[13] Zhang H, Chu X, Guo W, Wang S. Coexistence of Wi-Fi and heterogeneous small cell networks sharing unlicensed spectrum. IEEE Communications Magazine. 2015 Mar; 53(3): 158-64.

[14] Samarakoon S, Bennis M, Saad W, Debbah M, Latva-Aho M. Ultra dense small cell networks: Turning density into energy efficiency. IEEE Journal on Selected Areas in Communications. 2016 May; 34(5): 1267-80.

[15] Zhang H, Dong Y, Cheng J, Hossain MJ, Leung VC. Fronthauling for 5G LTE-U ultra dense cloud small cell networks. IEEE Wireless Communications. 2016 Dec; 23(6): 48-53.

[16] Dikmese S, Ilyas Z, Sofotasios PC, Renfors M, Valkama M. Sparse Frequency Domain Spectrum Sensing and Sharing Based on Cyclic Prefix Autocorrelation. IEEE Journal on Selected Areas in Communications. 2017 Jan; 35(1): 159-72.

[17] Zhang H, Jiang C, Beaulieu NC, Chu X, Wen X, Tao M. Resource allocation in spectrum-sharing OFDMA femtocells with heterogeneous services. IEEE Transactions on Communications. 2014 Jul; 62(7): 2366-77.

[18] Bennis M, Perlaza SM, Blasco P, Han Z, Poor HV. Self-organization in small cell networks: A reinforcement learning approach. IEEE transactions on wireless communications. 2013 Jul; 12(7): 3202-12.

[19] Zhang H, Jiang C, Beaulieu NC, Chu X, Wang X, Quek TQ. Resource allocation for cognitive small cell networks: A cooperative bargaining game theoretic approach. IEEE Transactions on Wireless Communications. 2015 Jun; 14(6): 3481-93.

[20] Kibria MG, Villardi GP, Nguyen K, Ishizu K, Kojima F. Heterogeneous Networks in Shared Spectrum Access Communications. IEEE Journal on Selected Areas in Communications. 2017 Jan; 35(1): 145-58.

[21] Zhang H, Nie Y, Cheng J, Leung VC, Nallanathan A. Sensing time optimization and power control for energy efficient cognitive small cell with imperfect hybrid spectrum sensing. IEEE Transactions on Wireless Communications. 2017 Feb; 16(2): 730-43. 
[22] Kulkarni K, Banerjee A. Adaptive transmission strategies to maximize packet throughput of cognitive radio under primary user queue stability constraint. In Signal Processing and Communications (SPCOM), 2014 International Conference on 2014 Jul 22 (pp. 1-6). IEEE.

[23] Dai J, Wang S. Clustering-Based Spectrum Sharing Strategy for Cognitive Radio Networks. IEEE Journal on Selected Areas in Communications. 2017 Jan; 35(1): 228-37.

[24] Duan R, Elmusrati M, Virrankoski R. Stable transmission for a cognitive-shared channel with rechargeable transmitters. InCommunications (ICC), 2012 IEEE International Conference on 2012 Jun 10 (pp. 4632-4636). IEEE.

[25] Simeone O, Bar-Ness Y, Spagnolini U. Stable throughput of cognitive radios with and without relaying capability. IEEE Transactions on Communications. 2007 Dec; 55(12): 2351-60.

[26] Zhang Q, Jia J, Zhang J. Cooperative relay to improve diversity in cognitive radio networks. IEEE Communications Magazine. 2009 Feb; 47(2): 111-7.

[27] Zou Y, Zhu J, Zheng B, Yao YD. An adaptive cooperation diversity scheme with best-relay selection in cognitive radio networks. IEEE transactions on signal processing. 2010 Oct; 58(10): 5438-45.

[28] Lee J, Wang H, Andrews JG, Hong D. Outage probability of cognitive relay networks with interference constraints. IEEE Transactions on Wireless Communications. 2011 Feb; 10(2): 390-5.

[29] Krikidis I, Devroye N, Thompson JS. Stability analysis for cognitive radio with multi-access primary transmission. IEEE Transactions on Wireless Communications. 2010 Jan; 9(1).

[30] Wang L, Fodor V. Dynamic cooperative secondary access in hierarchical spectrum sharing networks. IEEE Transactions on Wireless Communications. 2014 Nov; 13(11): 6068-80.

[31] Cheng SM, Ao WC, Tseng FM, Chen KC. Design and analysis of downlink spectrum sharing in two-tier cognitive femto networks. IEEE Transactions on Vehicular Technology. 2012 Jun; 61(5): 2194-207.

[32] Wang XY, Ho PH, Chen KC. Interference analysis and mitigation for cognitive-empowered femtocells through stochastic dual control. IEEE Transactions on Wireless Communications. 2012 Jun; 11(6): 2065-75.

[33] Xie R, Yu FR, Ji H, Li Y. Energy-efficient resource allocation for heterogeneous cognitive radio networks with femtocells. IEEE Transactions on Wireless Communications. 2012 Nov; 11(11): 3910-20.

[34] Le LB, Niyato D, Hossain E, Kim DI, Hoang DT. QoS-aware and energy-efficient resource management in OFDMA femtocells. IEEE Transactions on Wireless Communications. 2013 Jan; 12(1): 180-94.

[35] Omey, E. et S. Van Gulck (2008), "Central limit theorems for variances and correlation coefficients." http://www.edwardomey.com/nonsave/CLTforsandr.pdf

[36] López-Benítez M, Casadevall F. Improved energy detection spectrum sensing for cognitive radio. IET communications. 2012 May 22; 6(8): 785-96.

[37] Blumenfeld, D.: 'Operations research calculations handbook' (CRC Press, 2001)

[38] Liang YC, Zeng Y, Peh EC, Hoang AT. Sensing-throughput tradeoff for cognitive radio networks. IEEE transactions on Wireless Communications. 2008 Apr; 7(4): 1326-37.

[39] Urgaonkar R, Neely MJ. Opportunistic cooperation in cognitive femtocell networks. IEEE Journal on Selected Areas in Communications. 2012 Apr; 30(3): 607-16.

[40] Stotas S, Nallanathan A. On the outage capacity of sensing-enhanced spectrum sharing cognitive radio systems in fading channels. IEEE Transactions on communications. 2011 Oct; 59(10): 2871-82.

[41] Hong X, Wang J, Wang CX, Shi J. Cognitive radio in 5G: a perspective on energy-spectral efficiency trade-off. IEEE Communications Magazine. 2014 Jul; 52(7): 46-53.

[42] Ng DW, Lo ES, Schober R. Energy-efficient resource allocation in multi-cell OFDMA systems with limited backhaul capacity. IEEE Transactions on Wireless Communications. 2012 Oct; 11(10): 3618-31. 